\title{
Bohr Theory in the Atomic Physics
}

\author{
Haiyan Li \\ Department of Physics, Dezhou University, Dezhou 253023, China \\ Tel: 86-534-8985-879 E-mail: tianwaifeixian78@163.com
}

\begin{abstract}
Bohr Theory is one important stage in the development of the theory of atomic physics, and it has achieved great achievements when dealing with the problem of hydrogen atom and H-like ion, and it is on the important status in the teaching of atomic physics. Combining with teaching experiences, the historical background of Bohr Theory, three fundamental hypotheses of Bohr Theory, and the deduction and limitations of Bohr Theory are respectively introduced in this article.
\end{abstract}

Keywords: Bohr Theory, Quantization, Quantum mechanics

\section{Historical background of Bohr Theory}

At the beginning of 1900, Rutherford proposed the famous atomic nuclear-mode structure which was testified by the big angle $\alpha$-particle scattering experiment, but this model was acutely conflicted with the classical electromagnetic theory. On the one hand, this conflict is represented by that according to the classical electromagnetic theory, the electrons in the hydrogen atom will emit energies by the mode of electromagnetic wave when running around the nucleus which will finally induce the orbit radius smaller and smaller until it drops into the nucleus, and induce the collapse of atom, and that breaches the fact that the atom is quite stable. On the other hand, because the speed that the electron runs around the nucleus continuously change, so the wavelength of the atomic radiation will correspondingly continually change, and the spectrum emitted by the atom should be continuous spectrum, which is also conflicted with the fact that the atomic spectrum is the line spectrum in the experiment. To explain the experiment law of the hydrogen atom spectrum and overcome and the difficulties in the Rutherford atomic nuclear-model structure, Bohr who was only 28 years old bravely introduced the concept of quantum, which was proposed by Planck in 1900 and developed by Einstein in 1905, into the atom, and continually published three articles with tremendous meaning, i.e. well-known "The Great Trilogy" about the atom theory, which is a milestone in the modern physics. Bohr Theory created the new age of the atom physics, and it explained the spectrum feature of hydrogen atom which could not been explained in 30 years.

\section{2. "The Great trilogy" of Bohr Theory}

\subsection{Hypothesis of stationary state}

Bohr applied the nuclear-model structure model but breached the limitation of classical physics, and supposed the electron in the atom could only run on some special allowed orbit, and here, though the electron did the accelerated motion around the nucleus, it could not radiate the electromagnetic energy, so it would not drop into the nucleus because of the energy consumption, and be in the stable state. These states are called the stationary state of the atom. The stationary state energy of the atom only takes some discrete values E1, E2, E3, E4..., and these stationary state energies are called the energy level, and the orbit run by the electron when the atom is in the stationary state is called as the stationary state orbit.

\subsection{Condition of frequency}

Bohr made the analogy of the Planck's energy quanta hypothesis and Einstein's photons hypothesis, and according to the energy conversation law, the change of atom energy equaled to the energy that it emitted and absorbed photons. Bohr thought that when the atom transited between two stationary states, the absorbed or emitted radiation frequency $\gamma$ was exclusive, and the relationship between the energy difference and the frequency $\gamma$ in two stationary states was

$E_{n}-E_{m}=h v, E_{n}>E_{m}$

Here, Bohr introduced the Planck constant into the domain of atom. The so-called stationary sate is the non-substantive movement which only happens among stationary states. 


\subsection{Quantization of angular momentum}

According to the correspondence principle, Bohr thought of the quantization of corresponding orbit from the quantization of energy, and deduced the quantization of the angular momentum of orbit. He thought that only the orbit movement angular momentum of the out-nuclear electron L would satisfy

$L=m v r=n \frac{h}{2 \pi} \quad(\mathrm{n}$ is the quantum number, $1,2,3 \ldots)$,

the movement was stable, which is the selection rule to confirm the stationary state.

\section{Deduction of Bohr Theory}

\subsection{Quantization of orbit radius}

Suppose the out-nuclear electron in the hydrogen atom runs around the nucleus on certain orbit, and here the Coulomb force serves as the centripetal force

$m \frac{v^{2}}{r}=\frac{1}{4 \pi \varepsilon_{0}} \frac{e^{2}}{r^{2}}$

Introduce the quantization condition of angular momentum and eliminate $v$

$r=n^{2} \frac{\varepsilon_{0} h^{2}}{\pi m e^{2}}$

and suppose $a_{1}=\frac{\varepsilon_{0} h^{2}}{\pi m e^{2}}$

so, $r=n^{2} a_{1}$, which indicates that the electron orbit radius allowed in the hydrogen atom can only take a series of discrete values, not continual values.

\subsection{Quantization of energy}

The hydrogen atom energy is composed by the kinetic energy and the potential energy of the electron. Suppose the potential energy is 0 when $r=\infty$, the energy of the hydrogen atom is

$$
E_{n}=-\frac{1}{4 \pi \varepsilon_{0}} \frac{e^{2}}{2 r}
$$

Introduce the formula (3) into the formula (2)

and, $E_{n}=\frac{1}{n^{2}} \frac{m e^{2}}{8 \varepsilon_{0}{ }^{2} h^{2}}$

So the energy of the hydrogen atom is quantitative.

When $\mathrm{n}$ is large, the relatively change variables of the orbit radius and the orbit energy respectively are $\frac{\Delta r}{r} \approx 2 n$ and $\frac{\Delta E_{n}}{E_{n}} \approx 2 n$, so the orbit radius and the orbit energy can be regarded as continually changing when $\mathrm{n}$ is large. When $\mathrm{n}$ is very small, the electric orbit, the orbit radius and the orbit energy have no meanings in the quantum mechanics.

\subsection{Rydberg constant}

Introduce the formula (5) into the formula (1), and compare with the Rydberg formula $\widetilde{v}=R\left(\frac{1}{m^{2}}-\frac{1}{n^{2}}\right)$,

so $R=\frac{2}{\left(4 \pi \varepsilon_{0}\right)^{2}} \frac{m e^{4} \pi^{2}}{h^{3} c}$.

In 1914, Bohr considered that the difference between the computation value and the experiment value of Rydberg constant exceeded the experimental error, and he thought that the electron should run around the center of mass in the mutual function with the nuclear electron. According to the disposal of double-body problem, the Ryderg constant can 
be computed.

In the formula $R_{A}=\frac{2}{\left(4 \pi \varepsilon_{0}\right)^{2}} \frac{\mu e^{4} \pi^{2}}{h^{3} c}, \mu=\frac{M m}{M+m}$ is the discounted quality of the atom.

$R_{A}=\frac{1836}{1837} \times 1.097373143 \times 10^{-7} \mathrm{~m}^{-1}=1.0967758771 \times 10^{-7} \mathrm{~m}^{-1}$

Therefore, considering the effect of nuclear quality, the theoretical value and the experimental value of the Rydberg constant are basically consistent.

\section{Status of Bohr Theory}

Bohr Theory first opened out the experimental rule of the hydrogen atom spectrum theoretically, and one basic characterizes of the matters movement in the micro system, i.e. the quantization of the physical quantum. It inherited the past and forged ahead into the future in the transfer from the classical theory to the quanta theory. The more important is the hydrogen atom theory established by these ideas, which can better explain the experiment law of the hydrogen atom spectrum, and some quanta characters in the interior of the atom. For example, the quantitative stationary state of the atom and the frequency rule of the radiation were a leap in the process that people explored the micro structure in the interior of atom, and they established the base of the modern atom model, and were excessively accepted by people, so Bohr was praised as the forerunner of the modern microphysics.

The success of Bohr Theory is not only because that it can successfully explain the problem of hydrogen atom and H-like ion, but also embodied in following aspects.

(1) Bohr correctly pointed out the existence of the atom energy level, i.e. the energy of atom is quantitative, and it only took some certain discrete values, which was not only testified by the hydrogen atom and H-like ion, but also proved by the Frank-Hz experiment. That shows Bohr's hypothesis about the energy quantization has more general meaning than his theory of hydrogen atom.

(2) Bohr correctly proposed the concept of the stationary state, i.e. the atom in certain energy state $E_{n}$ didn't radiate the electromagnetic wave, and only when the atom transited from certain one energy state $E_{n}$ to another energy state $E_{m}$, it could emit the photons, and the frequency of photons is

$v=\frac{E_{m}-E_{n}}{h}$

The facts indicate that this conclusion is universally correct for various atoms, and his physical idea of the quantum jump has been accepted by the modern science.

(3) The angular momentum quantization derived from Bohr's quantization condition $\mathrm{L}=\mathrm{nh}$ is universally correct.

The hypothesis that the atom has the energy level and the hypothesis about the atom stability, the quantum jump and the angular momentum quantization are all disobedient to the classical theory. But just because of the jump of these concepts leaving form the classical idea, Bohr acquired the success. Bohr's this achievement helped people to know the world and established the base for the modern quantum theory. Therefore, Bohr's this theory is very meaningful in the history of physics.

Though Bohr Theory successfully explained the spectrum of the hydrogen atom and definitely pointed out the classical physics was inapplicable in the interior phenomena of the atom. But Bohr Theory has inevitable limitations. Its essential weakness is in its theoretical structure, and it is the integration of classical physics and the quantum concepts, and it is the half-classical and half-quantum theory. On the one hand, it admits that the classical mechanics and the classical electrodynamics would not be the same with the interior of the atom, and on the other hand, when Bohr studied the atom, he still regarded the electron as one classical particle, and continued to use the coordinate, speed, orbit and other concepts which were used to describe the macro phenomena in the classical mechanics. And it can not explain why the Coulomb interaction between the electron and nucleus in the atom would still be effective but the electromagnetic rule about the radiation frequency would lose the function. Bohr's quantum hypothesis lacked in necessary theoretical references, and though it was developed and extended, but it was changed essentially.

Furthermore, Bohr Theory can not explain the system such as the helium atom which only has more one electron than the electron, and it can not compute the energy level, the intension of spectral line, the polarization, the chromatic dispersion and the fine structure, and it can not explain how the atoms compose the molecule, liquids and solids, and it can not explain the non-periodic changing system. So Bohr Theory and the extended Bohr Theory, i.e. the Sommerfield Theory, are not thorough quantum theory, so it is called as the old quantum theory. Up to 1924, Debroglie proposed that the micro particles have the character of volatility, which was testified by the experiment, and then the nature of the 
micro particles was further known. The quantum mechanics based on that is the new quantum mechanics, hence the perfect theory about the atom phenomena formed. Still, Bohr Theory inherits the past and forges ahead into the future in the atom physics even in the modern physics. Just as someone visualized that "like a bridge, one end of the Bohr Theory is based on the classical concepts, and its another end leads people to the quantum world." Both its limitations and its advantages should be clearly realized, which could help researches to complete the transition form the classical concepts to the quantum concepts.

\section{References}

Chu, Shenglin. (1994). Atomic Physics. Beijing: China Higher Education Press.

Pei, Yongwei, Ji, Yanshen \& Song, Yali. (2004). More Discussion on the Establishment Process of Bohr Theory. Journal of Shenyang University. No.8.

Sun, Chunfeng \& Zheng, Dongmei. (2004). Compared Research of Bohr Theory: In Memory of the Born of Bohr Theory for 90 Anniversaries. Physics and Engineering. No.14 (1).

Wang, Fushan. (1986). Research on History of Modern Physics. Shanghai: Fudan University Press.

Yu, Shenqi, Long, Chunhua \& Liu, Huaxiang. (1990). Atomic Physics. Changchun: Northeast Normal University Press.

Zhou, Shaosen, Fan, Cheng \& Zhou, Jing. (1984). Atomic Physics. Shanghai: East China Normal University Press. 